\title{
Tick tock
}

\section{Thrombolysis \& acute management of ischemic stroke}

\author{
Alexander Levit (MD/PhD 2020), Brandon Chau (Meds 2018) \\ Faculty Reviewer: Dr Vladimir Hachinski, CM, MD, DSc, FRCPC (Department of Clinical Neurological Sciences)
}

\begin{abstract}
Ischemic stroke causes morbidity and death in $\mathbf{5 5 0 0 0}$ Canadians each year. While acute supportive therapy is essential for stabilizing ischemic stroke patients, resolution of cerebrovascular occlusion can only be accomplished by injection of thrombolytic agents. However, older guidelines restrict thrombolysis to within the first 3 hours of symptom onset. This short window of opportunity for thrombolytic treatment is complicated by its inherent adverse reactions, making it necessary to readily identify potential stroke cases, but also to diagnose accurately to avoid inappropriate treatment. In light of statistics that identify late presentation as the most common reason for stroke patients not to receive thrombolytic treatment, and meta-analyses that now show benefits of thrombolytic treatment up to 6 hours after stroke onset, guidelines are undergoing revision to allow larger treatment windows. This, along with continuing improvements in stroke treatment access and infrastructure, will hopefully lead to better outlooks for Canadians who suffer ischemic stroke.
\end{abstract}

It is not just a cliché: every minute really does count. On average, 1.9 million neurons, 14 billion synapses, and 12 kilometers of myelinated fibres are destroyed every 60 seconds following a large vessel ischemic stroke. Reminiscent of Christopher Nolan's Interstellar, the ischemic brain can age 3.6 years for every hour that a cerebral artery is left occluded. ${ }^{1}$ Unfortunately, this is not just fiction. Ischemic stroke is a leading cause of death and disability, a crippling reality for 55000 patients across Canada every year. ${ }^{2}$ While prevention is crucial, therapeutic options are especially effective when prompt thrombolytic intervention is possible. Given the time-sensitive nature of stroke management, it is important to be familiar with the general approach to acute stroke. We will focus on thrombolytic therapy for ischemic stroke but first, we will review the key points of stroke diagnosis.

\section{DIAGNOSIS}

History and physical examination offer vital information when an ischemic stroke is suspected. Usually, presentation will include a history of sudden onset focal neurological symptoms (Table), though symptoms may fluctuate in severity or worsen gradually. Headaches will occur in approximately 1 in 4 cases of stroke. For determining treatment options, the time of symptom onset is a critical feature of the history and should be detailed whenever possible. Ischemic stroke often occurs without pain, so many patients may not be woken by a stroke that occurs during sleep. In this case, it is assumed that the stroke began at the last time the patient was known to be symptom-free. Most presenting patients are alert, though infarction of the brainstem can cause a decreased level of consciousness. Nausea and vomiting may also occur if the brain stem is affected. ${ }^{3}$

As many as 1 out of 8 initial diagnoses of stroke may be incorrect, so it is important to consider all of the possible mimics. ${ }^{4,5}$ Many of these mimics will tend to have more global presentations than most strokes, although the opposite is sometimes true. ${ }^{3}$ For example, vertigo can often present with nausea and ataxia, mimicking the presentation of a stroke, particularly vertebrobasilar ischemia. ${ }^{4}$ However, vertebrobasilar ischemia is more likely to present with cranial nerve deficits, such as loss of corneal sting sensation and loss of nasal tickle, and other focal symptoms (eg diplopia, dysarthria, hemianopsia, transient global amnesia). One of the most common non-stroke referrals to stroke teams is syncope due to suspicion of brain stem ischemia, for which assessment for arrhythmias and excessive parasympathetic stimulation can rule out more common causes of syncope. Of all stroke mimics, unrecognized seizures followed by post-ictal paralysis or confusion may be the most common. ${ }^{4,5}$ Vigilance for a history of epilepsy and signs of seizure activity would direct appropriate investigations including electroencephalography. Other mimics include metabolic abnormalities (eg hypoglycemia, hepatic encephalopathy), drug overdose, neoplasia, and infection, which can be evaluated for with routine laboratory tests. ${ }^{3,4}$

Clinical evaluation can help differentiate hemorrhagic and ischemic strokes. Intracranial hemorrhage is more likely with the presence of coma on arrival, vomiting, severe headache, current anticoagulation therapy, hypertension, or non-diabetic hyperglycemia. However, imaging studies are still necessary to confidently rule out a hemorrhagic stroke or other vascular brain lesions. ${ }^{4}$ The current standard of imaging is noncontrast-enhanced CT, owing to the scan's speed, availability, and relatively low cost. The target timeframe for diagnosis of stroke patients is to complete CT examination within 25 minutes of arrival, with interpretation complete by the 45 minute mark from arrival. ${ }^{6}$ Laboratory tests should also be complete by this time and to ensure this kind of efficiency, stroke centres have to implement appropriate infrastructure.

\section{GENERAL SUPPORT \& THROMBOLYTIC THERAPY}

Acute ischemic stroke can lead to rapid patient destabilization, even without initial overt failure of vital function, so protective measures are imperative for the recovery of stroke patients. This includes monitoring oxygen saturation with a pulse oximeter and 
Table: Common patterns of focal neurological impairments correlated to region of infarct ${ }^{a}$

\begin{tabular}{|c|c|}
\hline Dominant (left) hemisphere & $\begin{array}{l}\text { - Aphasia } \\
\text { - Contralateral hemiparesis, sensory loss, } \\
\text { spatial neglect, homonymous hemianopia, } \\
\text { impaired conjugate gaze }\end{array}$ \\
\hline Nondominant hemisphere & $\begin{array}{l}\text { - Contralateral hemiparesis, sensory loss, } \\
\text { spatial neglect, homonymous hemianopia, } \\
\text { impaired conjugate gaze }\end{array}$ \\
\hline Subcortical \& brain stem & $\begin{array}{l}\text { - Unilateral hemiparesis, sensory loss } \\
\text { - Dysarthria } \\
\text { - Absent abnormalities of cognition, lan- } \\
\text { guage or vision }\end{array}$ \\
\hline Brain stem & $\begin{array}{l}\text { - Motor or sensory loss in all four limbs } \\
\text { - Crossed signs (ipsilateral cranial nerve \& } \\
\text { contralateral spinal deficits) } \\
\text { - Dysconjugate gaze } \\
\text { - Nystagmus } \\
\text { - Ataxia } \\
\text { - Dysarthria, dysphagia }\end{array}$ \\
\hline Cerebellum & $\begin{array}{l}\text { - Ipsilateral limb ataxia } \\
\text { - Gait ataxia }\end{array}$ \\
\hline
\end{tabular}

providing oxygen supplementation as needed to maintain $\geq 95 \%$ $\mathrm{O}_{2}$ saturation. Endotracheal intubation and careful monitoring of respiratory function may also be indicated if there is suspicion of brainstem ischemia or compression. ${ }^{7}$ Myocardial infarction and cardiac arrhythmias may develop secondary to stroke, and a 12 lead ECG should be monitored for all stroke patients. ${ }^{3}$ Fever development after stroke onset is a poor prognostic factor, and studies indicate that this should be treated with antipyretics and cooling devices. ${ }^{8}$ Induced hypothermia has been theorized to be neuroprotective, but evidence to support this approach is still indefinite. ${ }^{9,10,11}$

The specific goal for thrombolysis and subsequent brain tissue reperfusion is to salvage tissue in the ischemic penumbra-tissue surrounding the ischemic core that has not been yet been infarcted. ${ }^{3}$ This is accomplished with rapid intravenous administration of recombinant tissue plasminogen activator (rtPA), powerful thrombolytic agents that accelerate the breakdown of the fibrin meshwork of offending thromboemboli. Several drugs belong to the serine protease class of rtPA (alteplase, reteplase, tenecteplase), and all cleave endogenous plasminogen to its active form, plasmin, which degrades fibrin. With intravenous administration of thrombolytics, $37 \%$ of patients in Canadian hospitals achieve excellent clinical outcomes $(n=1135) .{ }^{12}$ In comparison, only $27 \%$ of ischemic stroke patients would be expected to achieve this level of recovery on standard therapy without thrombolysis. ${ }^{12,13}$ Excellent outcome was defined as a return to the level of function prior to stroke, as assessed by the modified Rankin Scale. Since these drugs are so effective at dissolving blood clots, they come with very serious risks of hemorrhage, including intracranial hemorrhage. This is especial- ly of concern with hemorrhagic stroke, but it is also a consideration in the treatment of ischemic stroke due to danger of conversion to intracranial hemorrhage. Understandably, active or recent bleeding are contraindications for thrombolytic therapy. Aspirin or other anticoagulants should not be given at the same time as thrombolytic therapy, as they increase the risk of bleeding. ${ }^{14}$

\section{LOOKING FORWARD}

Ongoing trials are investigating refinements to thrombolytic therapy, and the literature is very extensive and sometimes polarized. Many thrombolytics have been developed in an attempt to improve therapeutic benefit, to expand the window of opportunity for drug administration, and to reduce the rate of adverse events, namely hemorrhage. New drug candidates are often theorized to improve thrombolytic therapy because of increased biological halflife or improved target specificity. However, a Cochrane meta-analysis did not find adequate evidence to indicate any superior alternative to alteplase $(0.9 \mathrm{mg} / \mathrm{kg})$, the only FDA approved agent that is indicated for acute ischemic stroke. ${ }^{15}$ Similarly, intraarterial thrombolytic administration was theorized to reduce adverse events because of more localized delivery and lower therapeutic doses, but this was also found to be unsupported..$^{15}$ The potential advantage of intraarterial over intravenous administration may be offset by the delay in drug delivery because of relative procedural difficulty.

Although initial clinical trials on thrombolytic therapy found unfavourable outcomes in patients greater than 80 years of age and patients that have had symptoms for longer than 3 hours, more recent studies have had success with expanded inclusion criteria. The most recent Cochrane review suggests patients older than 80 years equally benefited from thrombolysis, and while treatment within 3 hours is still far more optimal in all age groups (OR 0.66, 95\% CI [0.56 - 0.79], $\mathrm{n}=2187)$, treatment within 6 hours still significantly reduced death or dependency (OR 0.84, 95\% CI [0.77 - 0.93], n = 6729). ${ }^{14}$ Unchanged since 2001, The Canadian Asssociation of Emergency Physicians still recommends rtPA treatment within 3 hours only, while the 2010 Canadian Best Practice Recommendations for Stroke Care indicate that rtPA can be given within 4.5 hours of symptom onset. ${ }^{16,17}$ Centres involved in thrombolysis protocol research may expand the window for treatment, so differences should be expected. London Health Sciences Centre currently offers rtPA treatment within 4.5 hours of symptom onset to patients of any age.

Despite the advances in patient outcomes, only $6.1 \%$ of ischemic stroke patients received thrombolysis throughout Canada's provinces in 2008-2009 ( $\mathrm{n}=9588)$. The territories were excluded in this study, leaving us only to speculate on the challenges for stroke management in these regions. Rates for utilization of thrombolytic agents were variable: comprehensive stroke centres treated 11\% of ischemic stroke cases with thrombolysis, compared to $5.7 \%$ in primary stroke centres, and just $1.0 \%$ in other centres. In this same study, the reasons documented for not treating with thrombolysis included patients presenting too late in $42 \%$ of cases, neurological deficit too mild in $24 \%$, clear medical contraindication in $5 \%$, and no reason was documented in $25 \%{ }^{18}$ While refining the eligibility criteria for rtPA treatment is important, these variable utilization 
rates indicate that serious infrastructure improvements will also have to be made in our ability to identify and respond to ischemic stroke events. With prompt identification and management, further improvements in thrombolysis, and national coordination of stroke care, we can look forward to better prognoses for acute ischemic stroke.

\section{REFERENCES}

1. Saver J. Time Is Brain--Quantified. Stroke. 2005;37(1):263-6.

2. Yip T, Demaerschalk B. Estimated cost savings of increased use of intravenous tissue plasminogen activator for acute ischemic stroke in Canada. Stroke. 2007;38(6):1952-5.

3. Adams H, Adams R, Brott T, del Zoppo G, Furlan A, Goldstein L, Grubb R, Higashida R, Kidwell C, Kwiatkowski T, Marler J, Hademenos G. Guidelines for the Early Management of Patients With Ischemic Stroke: A Scientific Statement From the Stroke Council of the American Stroke Association. Stroke. 2003;34(4):1056-83.

4. Spence J, Barnett H. Stroke prevention, treatment and rehabilitation. New York: McGraw-Hill Medical; 2012.

5. Norris J, Hachinski V. Misdiagnosis of stroke. Lancet. 1982;319(8267):328-31

6. Marler JR, Jones PW, Emr M. Proceedings of a national symposium on rapid identification and treatment of acute stroke; 1997; NIH publication No. 974239.

7. Treib J, Grauer MT, Woessner R, Morgenthaler M. Treatment of stroke on an intensive stroke unit: a novel concept. Intensive Care Med. 2000;26:1598-1611.

8. Hajat C, Hajat S, Sharma P. Effects of poststroke pyrexia on stroke outcome: a meta-analysis of studies in patients. Stroke. 2000;31:410-4.

9. Wu T, Grotta J. Hypothermia for acute ischaemic stroke. Lancet Neurol. 2013;12(3):275-84.

10. De Georgia M, Patel V. Critical care management in acute ischemic stroke. J Neurointerv Surg. 2010;3(1):34-7.

11. Den Hertog HM, van der Worp HB, Tseng M-C, Dippel DWJ. Cooling therapy for acute stroke. Cochrane Database Syst Rev. 2009

12. Hill M. Thrombolysis for acute ischemic stroke: results of the Canadian Alteplase for Stroke Effectiveness Study. CMAJ. 2005;172(10):1307-12.

13. Group T. Generalized efficacy of t-PA for acute stroke : Subgroup Analysis of the NINDS t-PA Stroke Trial. Stroke. 1997;28(11):2119-25.

14. Wardlaw J, Murray V, Berge E, del Zoppo G. Thrombolysis for acute ischaemic stroke. Cochrane Database Syst Rev. 2014

15. Wardlaw J, Koumellis P, Liu M. Thrombolysis (different doses, routes of administration and agents) for acute ischaemic stroke. Cochrane Database Syst Rev. 2013

16. Thrombolytic therapy for acute ischemic stroke (CAEP Position Statement) Can J Emerg Med. 2001;3(1):8-12.

17. Lindsay MP, Gubitz G, Bayley M, Hill MD, Davies-Schinkel C, Singh S, Phillips S. Canadian best practice recommendations for stroke care (Update 2010). On behalf of the Canadian Stroke Strategy Best Practices and Standards Writing Group. 2010; Ottawa, Ontario Canada: Canadian Stroke Network.

18. Ganesh A, Camden M, Lindsay P, Kapral M, Cote R, Fang J, Zagorski B, Hill $\mathrm{M}$. The quality of treatment of hyperacute ischemic stroke in Canada: a retrospective chart audit. CMAJ Open. 2014;2(4):E233-9.
19. Ouro-Bang’na Maman AF, Tomta K, Ahouangbévi S, et al. Deaths associated with anaesthesia in Togo, West Africa. Trop Doct. 2005 Oct;35(4):220-2.

20. Hodges SC, Mijumbi C, Okello M, et al. Anaethesia services in developing countries: defining the problems. Anaesthesia. 2007 Jan;62(1):4-11.

21. Cherian MN, Merry AF, Wilson IH. The World Health Organization and anaesthesia. Anaesthesia. 2007 Dec;62 Suppl 1:65-6.

22. Cotton M, Henry JA, Hasek L. Value innovation: an important aspect of global surgical care. Global Health. 2014 Jan;10(1):1

23. Disease Control Priorities Project. Promoting essential surgery in low-income countries: a hidden cost-effective treasure [Internet]. Washington, DC: Disease Control Priorities Project; 2008 [cited Mar 8 2014]. Available from: http://www.dcp2.org/file/158/dcpp-surgery.pdf

24. Dubowitz G, Breyer K, Lipnick M, et al. Accuracy of the Lifebox pulse oximeter during hypoxia in healthy volunteers. Anaesthesia. 2013 Dec;68(12):1220-3

\section{Nidin \\ Labrador-Grenfell Health \\ EMPLOYMENT OPPORTUNITIES}

Interested in working in Northern Newfoundland and Labrador? Then you may be the type of physician that Labrador-Grenfell Health is seeking to join our multi-disciplinary health care team which provides integrated health care and emphasizes health promotion and disease prevention.

We are currently recruiting for Medical Internists, General Surgeons, Pediatricians, Obstetrician/Gynecologists, Anesthetists and Family Physicians.

Candidates must be eligible for full or provisional licensure by the College of Physicians and Surgeons of Newfoundland and Labrador. For a preliminary review of your $\mathrm{CV}$ for licensure, please submit using the format provided by the College at www.cpsnl.ca. Physicians are eligible for a non-pensionable retention incentive which is paid after one year of service and increases as the period of service continues up to three years. Additional remuneration is also available for coverage of extra call. We offer an accredited CME program and an allowance for Continuing Medical Educational activities. We also assist with relocation.

The LGRHA region offers an abundance of outdoor activities, spectacular scenery and wildlife and a very safe family environment. If you are interested in one of these demanding yet rewarding positions and feel that you have the skills required to work in this challenging environment please contact:

Dr. Kweku Dankwa, Associate VP, Medical Services Angie Elliott, Manager, Medical Services Labrador-Grenfell Regional Health Authority

178-200 West Street • St. Anthony, NL • Canada - AOK 4SO T: 709-454-0127 • F: 709-454-2052 • www.lghealth.ca

Email: kweku.dankwa@lghealth.ca or angela.elliott@lghealth.ca 